\title{
Effects of Dipeptidyl Peptidase-4 Inhibitors on Renal Outcomes in Patients with Type 2 Diabetes: A Systematic Review and Meta-Analysis
}

\author{
Jae Hyun Bae ${ }^{1, *}$, Sunhee Kim²,*, Eun-Gee Park ${ }^{2}$, Sin Gon Kim ${ }^{1}$, Seokyung Hahn ${ }^{3,4}$, Nam Hoon Kim ${ }^{1}$ \\ ${ }^{1}$ Department of Internal Medicine, Korea University College of Medicine; ${ }^{2}$ Interdisciplinary Program in Medical Informatics, \\ Seoul National University College of Medicine; ${ }^{3}$ Division of Medical Statistics, Medical Research Collaborating Center, Seoul \\ National University Hospital; ${ }^{4}$ Department of Medicine, Seoul National University College of Medicine, Seoul, Korea
}

Background: To investigate the effects of dipeptidyl peptidase-4 (DPP-4) inhibitors on renal outcomes in patients with type 2 diabetes. Methods: MEDLINE, Embase, and the Cochrane Central Register of Controlled Trials were searched to identify randomized controlled trials (RCTs) of DPP-4 inhibitors from inception to September 2017. We selected eligible RCTs comparing DPP-4 inhibitors with placebo or other antidiabetic agents and reporting at least one renal outcome. A meta-analysis was conducted to calculate standardized mean differences, weighted mean differences (WMDs), relative risks (RRs), and 95\% confidence intervals (CIs) for each renal outcome.

Results: We included 23 RCTs with 19 publications involving 41,359 patients. Overall changes in urine albumin-to-creatinine ratio were comparable between DPP-4 inhibitors and controls $(P=0.150)$. However, DPP-4 inhibitors were associated with significantly lower risk of incident microalbuminuria (RR, $0.89 ; 95 \% \mathrm{CI}, 0.80$ to $0.98 ; P=0.022)$ and macroalbuminuria (RR, $0.77 ; 95 \% \mathrm{CI}, 0.61$ to $0.97 ; P=0.027$ ), as well as higher rates of regression of albuminuria (RR, $1.22 ; 95 \% \mathrm{CI}, 1.10$ to $1.35 ; P<0.001)$ compared with controls. Although DPP-4 inhibitors were associated with small but significantly lower estimated glomerular filtration rate (WMD, $-1.11 \mathrm{~mL} / \mathrm{min} / 1.73 \mathrm{~m}^{2} ; 95 \% \mathrm{CI},-1.78$ to $\left.-0.44 ; P=0.001\right)$, there was no difference in the risk of end-stage renal disease between two groups (RR, $0.93 ; 95 \% \mathrm{CI}, 0.76$ to $1.14 ; P=0.475$ ).

Conclusion: DPP-4 inhibitors had beneficial renal effects mainly by reducing the risk of development or progression of albuminuria compared with placebo or other antidiabetic agents.

Keywords: Albuminuria; Diabetes complications; Diabetes mellitus, type 2; Diabetic nephropathies; Dipeptidyl-peptidase IV inhibitors; Glomerular filtration rate; Kidney failure, chronic; Meta-analysis; Systematic review

Received: 14 December 2018, Revised: 3 February 2019,

Accepted: 26 February 2019

Corresponding authors: Nam Hoon Kim

Department of Internal Medicine, Korea University College of Medicine,

73 Inchon-ro, Seongbuk-gu, Seoul 02841, Korea

Tel: +82-2-920-6268, Fax: +82-2-953-9355, E-mail: pourlife@korea.ac.kr

Seokyung Hahn

Department of Medicine, Seoul National University College of Medicine,

103 Daehak-ro, Jongno-gu, Seoul 03080, Korea

Tel: +82-2-740-8911, Fax: +82-2-743-8361, E-mail: hahns@snu.ac.kr

*These authors contributed equally to this work.

\section{Copyright $\odot 2019$ Korean Endocrine Society}

This is an Open Access article distributed under the terms of the Creative Commons Attribution Non-Commercial License (http://creativecommons.org/ licenses/by-nc/4.0/) which permits unrestricted non-commercial use, distribution, and reproduction in any medium, provided the original work is properly cited. 


\section{INTRODUCTION}

Diabetic kidney disease (DKD) is one of the major microvascular complications of type 2 diabetes and a leading cause of endstage renal disease (ESRD) accounting for one-third of all patients initiating renal replacement therapy (RRT) worldwide $[1,2]$. DKD is clinically characterized by persistent albuminuria, progressive decline in renal function, and the development of ESRD [3]. In the development or progression of DKD, prolonged hyperglycemia plays a crucial role through an increase in intracellular glucose metabolism, advanced glycation end products, oxidative stress, and epigenetic reprogramming [4]. In this regard, intensive glucose control has been proven to be effective in preventing or delaying the development and progression of DKD [5,6]. However, the effects of individual antidiabetic drugs on the kidney have been reported to be different [7].

Dipeptidyl peptidase-4 (DPP-4) inhibitors are a class of antidiabetic agents that potentiate incretin action by reducing degradation of glucagon-like peptide-1 and glucose-dependent insulinotropic polypeptide [8]. DPP-4 inhibitors have been used safely in patients with chronic kidney disease, but their effects on renal outcomes are uncertain. Preclinical studies suggested pleiotropic effects of DPP-4 inhibition which might be beneficial to the kidney [8-10]. However, in clinical trials, the effects of DPP-4 inhibitors on DKD were inconsistent. In the Saxagliptin Assessment of Vascular Outcomes Recorded in Patients with Diabetes Mellitus (SAVOR)-Thrombolysis in Myocardial Infarction (TIMI) 53 trial, saxagliptin significantly reduced urine albumin-to-creatinine ratio (UACR) without affecting renal function [11]. On the other hand, in the Trial Evaluating Cardiovascular Outcomes with Sitagliptin (TECOS) trial, sitagliptin showed a small but early decline in estimated glomerular filtration rate (eGFR) which lasted for 48 months with no difference in changes of UACR [12]. It also should be noted that in those studies renal outcomes were neither primary nor secondary endpoints $[13,14]$. In a study to assess albuminuria-lowering effect as a key efficacy outcome, linagliptin did not have beneficial effects on either albuminuria or renal function [15]. However, generalization of these results would be cautious in that the study comprised patients with relatively early stage of DKD. Indeed, in the SAVOR-TIMI 53 trial, the decrease in albuminuria was greater in patients with lower eGFR [11]. Therefore, to clarify the renal effects of DPP-4 inhibitors, additional method is needed to integrate the results and complement the limitations of individual randomized controlled trials (RCTs), even if they did not initially evaluate renal outcomes.
In the present study, we performed a systematic review and meta-analysis of RCTs to investigate the effects of DPP-4 inhibitors on individual renal outcomes including ESRD compared with placebo or other antidiabetic agents in patients with type 2 diabetes.

\section{METHODS}

We conducted the systematic review and meta-analysis based on a prespecified protocol developed by authors (Appendix 1) and reported the results according to the Preferred Reporting Items for Systematic Review and Meta-Analysis (PRISMA) statement (Supplemental Table S1) [16].

\section{Search strategy and study selection}

We searched MEDLINE, Embase, and the Cochrane Central Register of Controlled Trials to identify relevant RCTs published in full-text articles from inception to September 2017 with no restriction of language. The search terms used for DPP4 inhibitors were DPP-4 inhibitor or DPP-4 inhibitor or alogliptin or anagliptin or evogliptin or gemigliptin or linagliptin or omarigliptin or saxagliptin or sitagliptin or teneligliptin or trelagliptin or vildagliptin.

We included RCTs comparing DPP-4 inhibitors with placebo or other antidiabetic agents in patients with type 2 diabetes. The eligible studies were at least 12 weeks of study duration and reported one or more renal outcomes including changes in UACR or eGFR, and the development of microalbuminuria, macroalbuminuria, doubling of serum creatinine levels, renal failure, ESRD, RRT, dialysis, or kidney transplantation. Pooled analysis for multiple RCTs was also included when it provided additional information on renal outcomes which had not been reported in the original publications. After removing duplicate publications, we screened titles and abstracts, and thoroughly reviewed full-text articles according to inclusion and exclusion criteria.

\section{Data extraction}

Two authors (S.K. and N.H.K.) independently extracted data from included studies according to the standardized protocol (Appendix 1). Prespecified outcomes of interests were changes in UACR and eGFR, the development of microalbuminuria (UACR $>30 \mathrm{mg} / \mathrm{g}$ ) and macroalbuminuria (UACR $>300 \mathrm{mg} / \mathrm{g}$ ), regression of albuminuria (defined as the development of normoalbuminuria from microalbuminuria or macroalbuminuria; development of microalbuminuria from macroalbuminuria), and incident ESRD. Any discrepancies were resolved by con- 
sensus among the authors (S.K., N.H.K., and S.H.). For continuous variables, mean changes from baseline and their standard deviations were extracted from both intervention (DPP-4 inhibitors) and control (placebo or other antidiabetic agents) groups as summary measures. In a study which reported changes in UACR according to eGFR categories [11], we extracted pooled mean differences between groups. When only mean difference between groups and its $P$ value was reported, we derived the standard error of the mean difference from the test statistic [17]. For dichotomous variables, we extracted the number of patients reporting each renal outcome. In addition, we obtained information about the first author, publication year, number and mean age of randomized participants, duration of follow-up, intervention and comparison treatment, and background antidiabetic agents. For multiple-dose studies, we combined the different dose groups of the same drug into a single group. For three-arm studies, we split a common arm into two groups and considered each pairwise comparison separately.

\section{Assessment of study quality and risk of bias}

The Cochrane Risk of Bias Tool was used to assess study quality and risk of bias. Two independent authors (S.K. and N.H.K.) reviewed included studies and judged the risk of bias as adequate (low risk of bias), unclear (unclear risk of bias), or inadequate (high risk of bias) from each of six domains: sequence generation, allocation concealment, blinding, incomplete outcome data, selective reporting, and other sources of bias [18]. Any discrepancies were resolved by the authors (S.K., N.H.K., and S.H.).

\section{Statistical analysis}

Standardized mean differences (SMDs) were used for changes in UACR since the results were reported in different scales for this outcome [19]. Hedges' $g$ was used for the estimate of SMDs to take the small number of studies into account [20]. Weighted mean differences (WMDs) and 95\% confidence intervals (CIs) were calculated for changes in eGFR. The SMDs were additionally considered for changes in eGFR. Relative risks (RRs) and 95\% CIs were calculated to assess effect size for the development of microalbuminuria and macroalbuminuria, regression of albuminuria, and incident ESRD. In the meta-analysis, we calculated a combined estimate and its $95 \%$ CI using a random-effects model. Cochran's Q test was used for testing statistical heterogeneity and the $I^{2}$ statistic was used to measure the degree of heterogeneity [19]. The $I^{2}$ statistics of $0 \%$ to $40 \%, 30 \%$ to $60 \%, 50 \%$ to $90 \%$, and $75 \%$ to $100 \%$ was regarded as not im- portant, moderate, substantial, and considerable heterogeneity, respectively [19]. We used the funnel plot and Egger's test to address reporting bias for the renal outcome with sufficient number of studies for the exploration $[21,22]$. In prespecified subgroup analysis, we evaluated changes in UACR according to sodium-glucose cotransporter 2 (SGLT2) inhibitors versus nonSGLT2 inhibitors in the control group. All statistical analyses were conducted using STATA version 12 (Stata Corp LLC, College Station, TX, USA). For treatment effects and test for heterogeneity, $P<0.05$ and $P<0.10$ were regarded as statistically significant, respectively.

\section{Ethical approval}

This article does not contain examinations performed on human participants in that ethical approval is not necessary.

\section{RESULTS}

\section{Characteristics of included studies}

Fig. 1 shows study screening and selection process. Of 5,562 records identified through electronic database search, we included 23 eligible RCTs with 19 publications in the analysis. Two publications $[12,14]$ reported different outcomes from the
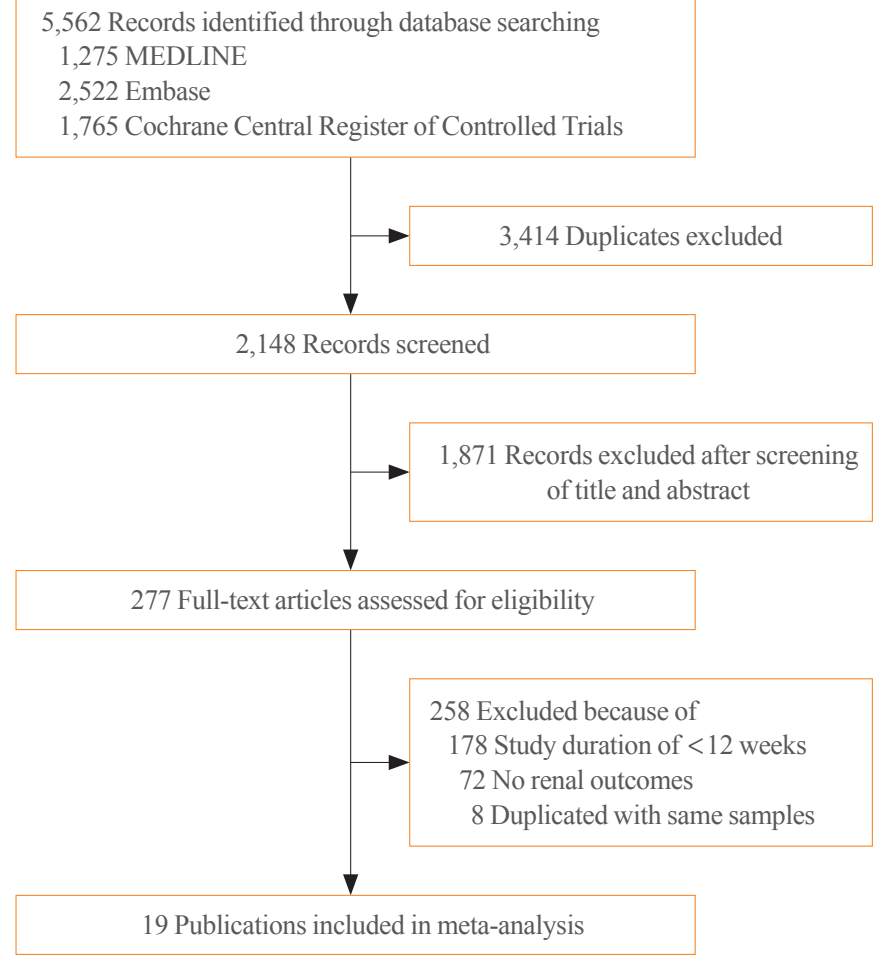

Fig. 1. Flow diagram of study screening and selection process. 
same study [12] and both were included in the analysis. One publication [23] was a pooled analysis of four RCTs [24-27], and another one study was an open-label, prospective RCT [28]. DPP-4 inhibitors were not confined to a specific agent in one study as they were used as a control [29]. Characteristics of the studies are presented in Table 1 [11,12,14,15,23,28-41]. A total of 41,359 participants were randomly assigned to either intervention or control group. The number of participants in individual studies ranged from 36 to 16,492 . The study duration of two studies lasted up to 4 years $[12,14]$, and one study reported results with a median duration of 2.1 years [11]. The remaining studies had 12 to 160 weeks of study duration. Baseline eGFR of participants was $\geq 60 \mathrm{~mL} / \mathrm{min} / 1.73 \mathrm{~m}^{2}$ in five studies [30-34] and $\geq 30 \mathrm{~mL} / \mathrm{min} / 1.73 \mathrm{~m}^{2}$ in five studies [12,14,15,23,35]. Two studies did not describe inclusion or exclusion criteria for baseline eGFR or serum creatinine levels $[36,37]$.

\section{Assessment of study quality and risk of bias}

Supplemental Fig. S1 summarizes the risk of bias assessment. We evaluated 23 RCTs published in 19 publications (Supplemental Table S2). Fourteen studies reported adequate random sequence generation and adequate allocation concealment. Nine studies did not state sequence generation [23,24,26-29,37-39] and allocation concealment [23,24,26-28,36-39]. Twenty-two studies reported adequate blinding of participants and personnel. One study was an open-label RCT [28], but classified as reporting adequate blinding because only objective findings were included in our study. One study did not describe blinding method [38]. Thirteen studies were judged as adequate for completeness of outcome data. Two studies did not clarify the number of participants in the analysis [23,37], and eight studies reported incomplete outcome data because of losses to follow-up $[11,24,26,27,29,30,33,38]$. Four studies [24-27] were integrated into one publication as a pooled analysis [23] and there was the possibility of selective reporting.

\section{Changes in UACR and eGFR}

Fig. 2 shows changes in UACR of SGLT2 inhibitors compared with those of control. Overall changes in UACR were not significantly different between groups $(P=0.150)$. The test for heterogeneity showed substantial heterogeneity across the studies $\left(I^{2}=67.9 \%, P=0.002\right)$, and this heterogeneity contributed to asymmetry in the funnel plot (Supplemental Fig. S2A). However, in the subgroup analysis according to the antidiabetic agent in the control group, DPP-4 inhibitors significantly lowered UACR compared with non-SGLT2 inhibitors $(P=0.019)$.
Meanwhile, DPP-4 inhibitors insignificantly increased UACR compared with SGLT2 inhibitors $(P=0.389)$. This difference in UACR changes was not statistically significant between the two subgroups $(P=0.105)$. Fig. 3 shows the comparison of changes in eGFR between DPP-4 inhibitors and controls. DPP-4 inhibitors showed a small but significant decline in eGFR compared with controls ([WMD, $-1.11 \mathrm{~mL} / \mathrm{min} / 1.73 \mathrm{~m}^{2} ; 95 \% \mathrm{CI},-1.78$ to $-0.44 ; P=0.001$ ], [SMD, $-0.07 ; 95 \% \mathrm{CI},-0.12$ to $-0.02 ; P=$ $0.009])$. The test for heterogeneity showed moderate heterogeneity across the studies $\left(I^{2}=40.5 \%, P=0.064\right.$ on the test of WMD; $I^{2}=43.2 \%, P=0.048$ on the test of SMD). The funnel plot was symmetrical, and Egger's test gave a $P$ value of 0.191 (Supplemental Fig. S2B).

\section{Development, progression, and regression of albuminuria} DPP-4 inhibitors significantly reduced the risk of developing microalbuminuria (RR, $0.89 ; 95 \% \mathrm{CI}, 0.80$ to $0.98 ; P=0.022$ ) and macroalbuminuria (RR, $0.77 ; 95 \% \mathrm{CI}, 0.61$ to $0.97 ; P=$ 0.027) compared with controls. However, the effects of DPP-4 inhibitors on incident albuminuria were mainly driven by one large trial (Supplemental Fig. S3) [11]. There was no heterogeneity across the studies on both microalbuminuria $\left(I^{2}=0.0 \%\right.$, $P=0.471)$ and macroalbuminuria $\left(I^{2}=1.3 \%, P=0.363\right)$ (Fig. $4 \mathrm{~A}, \mathrm{~B})$. In addition, DPP-4 inhibitors were associated with significantly higher rates of regression of albuminuria compared with controls (RR, 1.22; 95\% CI, 1.10 to $1.35 ; P<0.001$ ) (Fig. $4 \mathrm{C})$. There was no heterogeneity across the studies $\left(I^{2}=0.0 \%\right.$, $P=0.439$ ). The tests for funnel plot asymmetry were not performed on albuminuria because of the small number of studies $[11,30,32]$.

\section{Development of ESRD}

DPP-4 inhibitors did not reduce the risk of developing ESRD in patients with type 2 diabetes compared with controls (RR, 0.93; 95\% CI, 0.76 to $1.14 ; P=0.475$ ) (Fig. 4D). There was no heterogeneity across the studies $\left(I^{2}=0.0 \%, P=0.853\right)$. Asymmetry in the funnel plot was not evaluated due to the small number of studies $[11,30,32]$.

\section{DISCUSSION}

In this systematic review and meta-analysis, we found that DPP-4 inhibitors had beneficial effects on renal outcomes mainly by reducing albuminuria compared to placebo or other antidiabetic agents in patients with type 2 diabetes. On the other hand, DPP-4 inhibitors treatment was associated with a small decline 


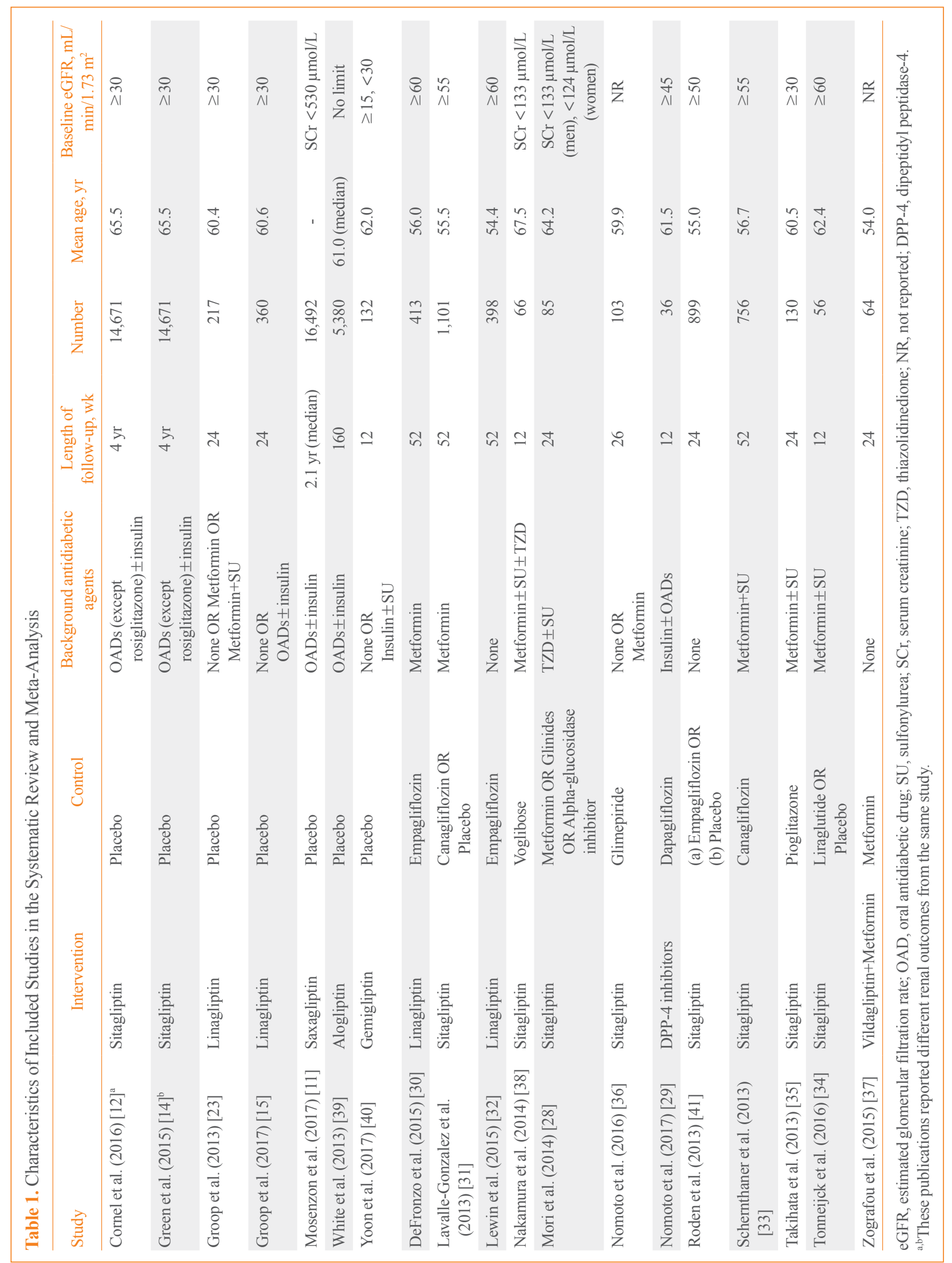




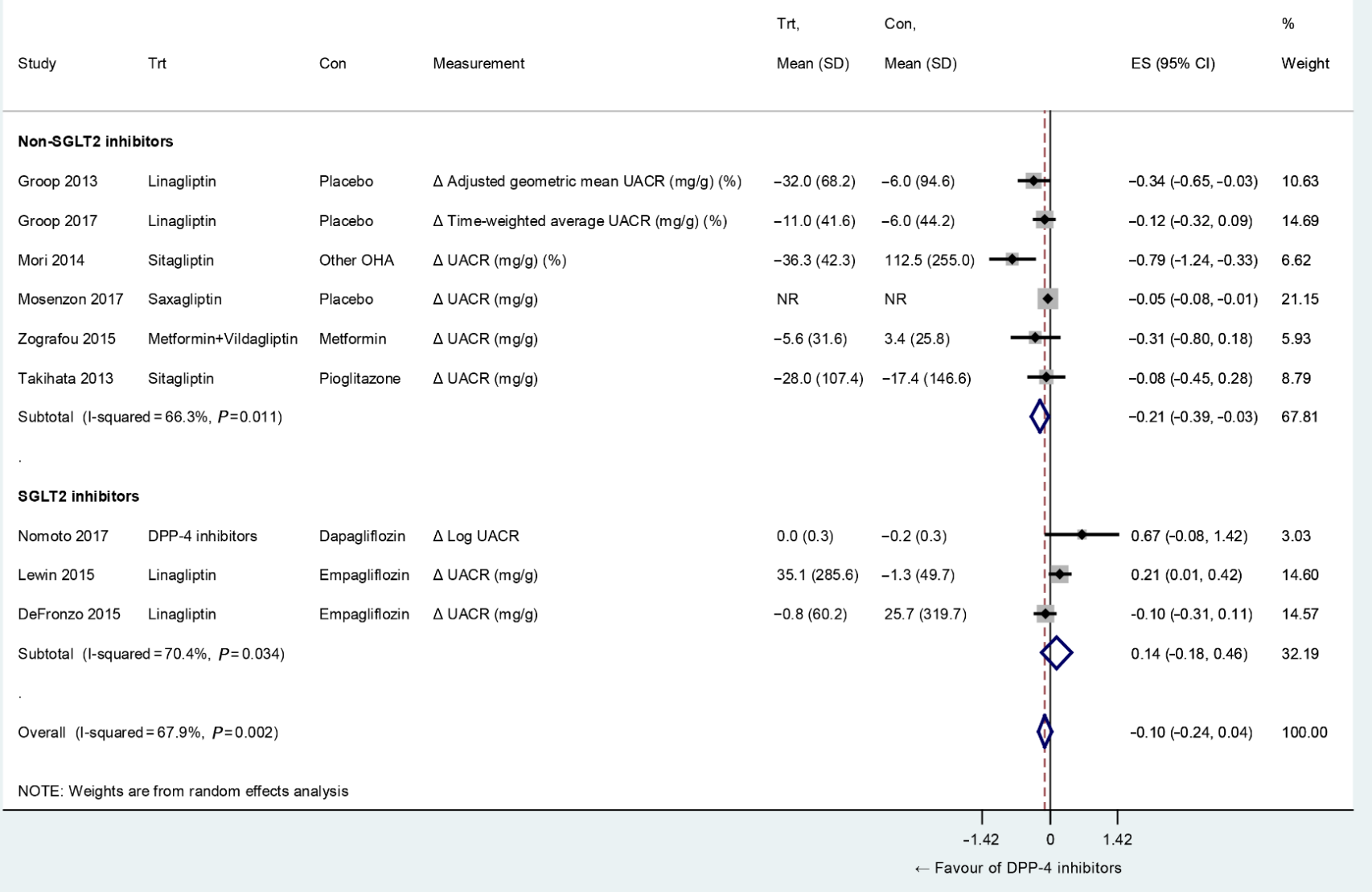

Fig. 2. Standardized mean differences in changes in urine albumin-to-creatinine ratio (UACR) from baseline (mg/g) for dipeptidyl peptidase-4 (DPP-4) inhibitors versus placebo or other antidiabetic agents, according to non-sodium-glucose cotransporter 2 inhibitors and sodium-glucose cotransporter 2 inhibitors in the control group. Trt, treatment group; Con, control group; SD, standard deviation; ES, effect size; CI, confidence interval; SGLT2, sodium-glucose cotransporter 2; OHA, oral hypoglycemic agent; NR, not reported.

in eGFR compared with controls. The risk of ESRD was not different between groups.

The beneficial effects of DPP-4 inhibitors on albuminuria were consistently demonstrated by reducing the risk of development or progression of both microalbuminuria and macroalbuminuria. These favorable effects were largely driven by the SAVOR-TIMI 53 trial [11]. However, decreases in UACR observed in other clinical trials $[23,42]$ supported albuminurialowering effects of DPP-4 inhibitors in our meta-analysis. There are several lines of evidence that may explain the mechanism by which DPP-4 inhibitors improve albuminuria. Preclinical studies showed that DPP-4 inhibitors were beneficial to DKD by reducing oxidative stress, inflammation, and histopathologic changes in renal injury [43-46]. Interestingly, linagliptin ameliorated kidney fibrosis in streptozotocin-induced diabetic mice without altering blood glucose levels by inhibition of endotheli- al-to-mesenchymal transition and restoration of microRNA 29s [47]. An increase in stromal cell-derived factor- $1 \alpha$ also contributed to antioxidative and antifibrotic effects of DPP-4 inhibitors [48]. Additionally, DPP-4 inhibitors induced natriuresis in patients with type 2 diabetes [49]. However, they exerted natriuretic effects mainly on distal renal tubule, not on proximal tubule as in SGLT2 inhibitors [49,50]. Moreover, natriuretic response to DPP-4 inhibitor was blunted in diabetic mice [51]. Hence, DPP-4 inhibitors could reduce albuminuria through antioxidant, anti-inflammatory, and anti-fibrotic effects without significant changes in renal hemodynamics.

Intriguingly, the direction of treatment effects of DPP-4 inhibitors was different according to the antidiabetic agent in the control group. DPP-4 inhibitors significantly reduced UACR compared with controls other than SGLT2 inhibitors, but they had a tendency to increase UACR when compared with SGLT2 


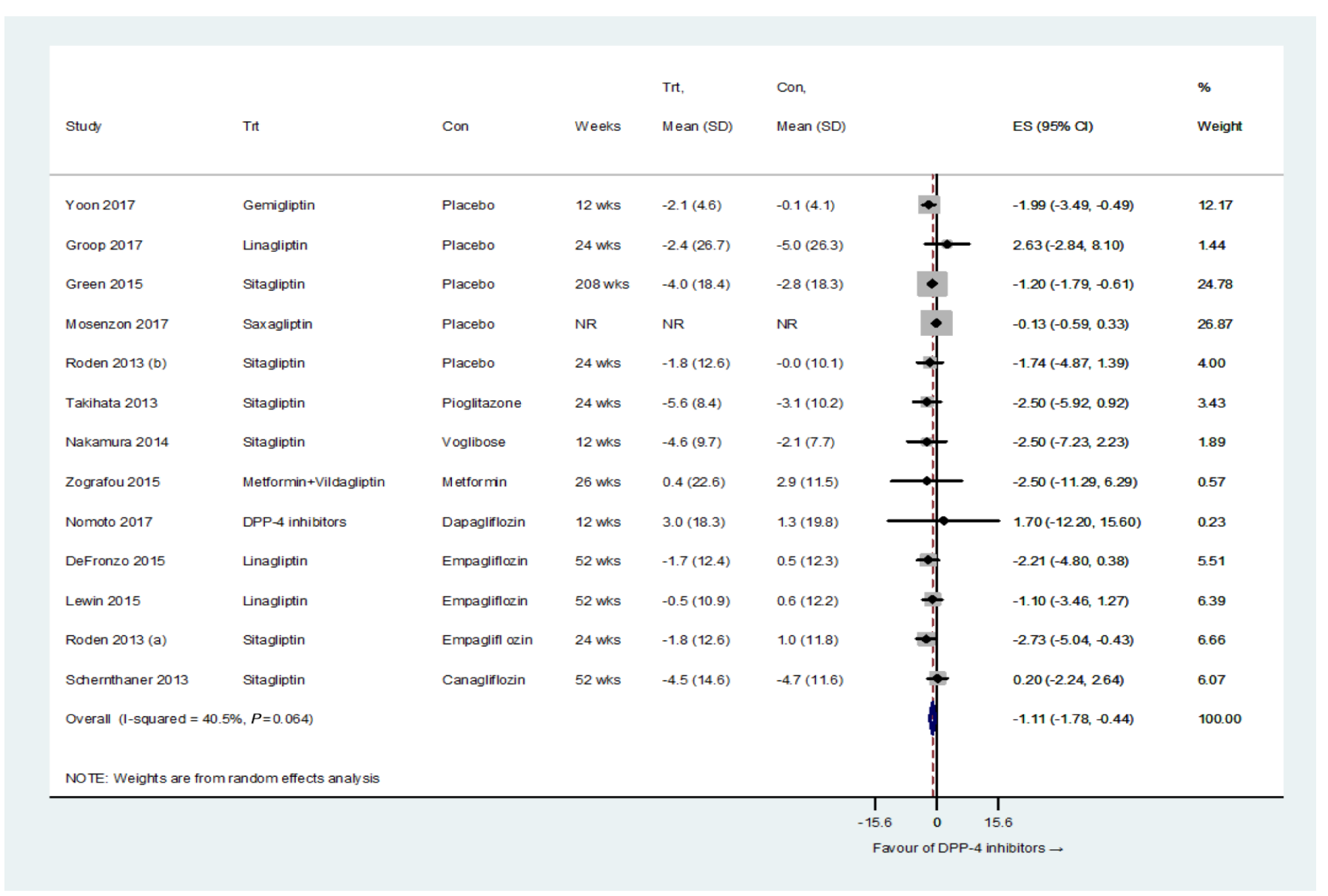

Fig. 3. Weighted mean differences in changes in estimated glomerular filtration rate from baseline ( $\left.\mathrm{mL} / \mathrm{min} / 1.73 \mathrm{~m}{ }^{2}\right)$ for dipeptidyl peptidase-4 (DPP-4) inhibitors versus placebo or other antidiabetic agents. Trt, treatment group; Con, control group; SD, standard deviation; ES, effect size; CI, confidence interval; NR, not reported.

inhibitors. In the development of microalbuminuria and macroalbuminuria, the direction of treatment effects was consistent with changes in UACR, although it was limited due to the small number of studies. These findings suggest that SGLT2 inhibitors might be more effective than DPP-4 inhibitors in reducing albuminuria, which requires further investigation.

We also found that DPP-4 inhibitors marginally decreased eGFR compared with controls. A small decline in eGFR after DPP-4 inhibitor treatment has already been recognized in the TECOS trial [12]. However, it is uncertain that these small changes in eGFR have any clinical implications. Moreover, most of the studies in our meta-analysis had less than 52 weeks of follow-up, which was limited to observe their long-term effects. Different from changes in renal function, DPP-4 inhibitors tended to reduce the risk of ESRD compared with control in the pooled analysis of three large cardiovascular outcome trials, which might suggest favorable effects on the decline in renal function $[11,14,39]$. In terms of reduction in the risk of ESRD, it has been suggested that DPP-4 inhibitors exerted beneficial effects on multiple risk factors for DKD including albuminuria $[11,23,28]$, inflammation [52-54], and endothelial dysfunction $[38,55]$ besides glucose-lowering effects.

In line with our findings, the Cardiovascular Renal Microvascular Outcomes with Linagliptin in Patients with Type 2 Diabetes Mellitus (CARMELINA) study group recently announced that linagliptin significantly reduced the risk of albuminuria progression without affecting other renal outcomes including ESRD. The detailed results to be published soon are expected to help understand better the renal effects of DPP-4 inhibitors.

There are several limitations in the present study. First, renal outcomes were not evaluated as prespecified endpoints in most studies included in our meta-analysis. Second, about $40 \%$ of included studies had the possibility of selection bias or attrition bias. Third, the number of studies included in the analysis of in- 

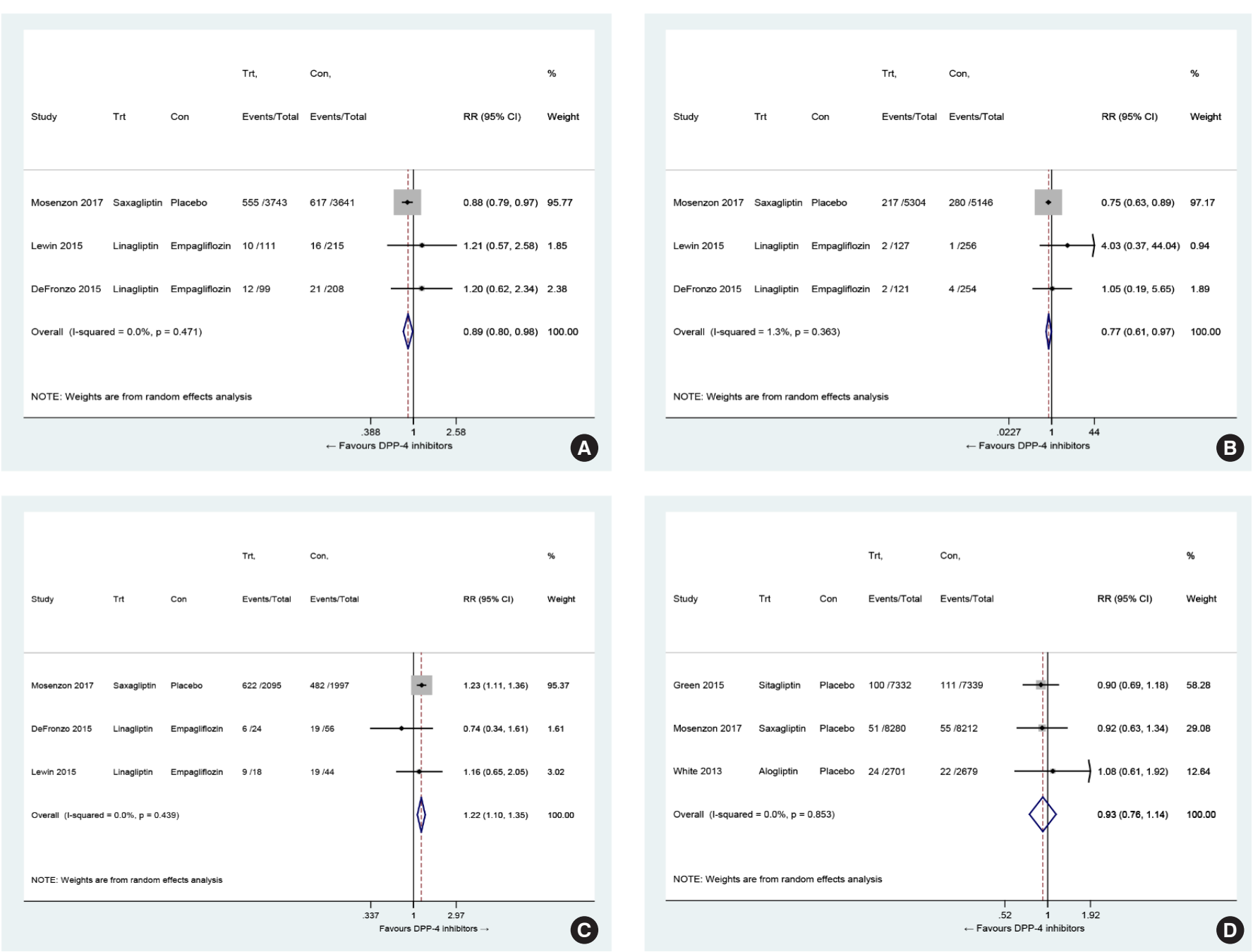

Fig. 4. Relative risks of incident albuminuria, regression of albuminuria, and end-stage renal disease for dipeptidyl peptidase-4 (DPP-4) inhibitors versus placebo or other antidiabetic agents. (A) Microalbuminuria, (B) macroalbuminuria, (C) regression of albuminuria, and (D) end-stage renal disease. Trt, treatment group; Con, control group; RR, relative risk; CI, confidence interval.

cident albuminuria or ESRD are relatively small. Thus, the results of our meta-analysis should be interpreted cautiously.

In conclusion, our systematic review and meta-analysis demonstrated that DPP-4 inhibitors had renoprotective effects by reducing the risk of development or progression of albuminuria without affecting the risk of ESRD in patients with type 2 diabetes compared with placebo or other antidiabetic agents.

\section{CONFLICTS OF INTEREST}

No potential conflict of interest relevant to this article was reported.

\section{ACKNOWLEDGMENTS}

This study was supported by the Korean Endocrine Society of EnM Research Award 2017.

\section{AUTHOR CONTRIBUTIONS}

Conception or design: J.H.B., S.K., E.G.P., S.G.K., S.H., N.H.K. Acquisition, analysis, or interpretation of data: J.H.B., S.K., E.G.P., S.H., N.H.K. Drafting the work or revising: J.H.B., S.K., S.H., N.H.K. Final approval of the manuscript: J.H.B., S.K., E.G.P., S.G.K., S.H., N.H.K. Obtained funding, statistical analysis: S.K., S.H., N.H.K. 


\section{ORCID}

Jae Hyun Bae https://orcid.org/0000-0002-1384-6123

Seokyung Hahn https://orcid.org/0000-0002-4684-4917

Nam Hoon Kim https://orcid.org/0000-0002-9926-1344

\section{REFERENCES}

1. Zelnick LR, Weiss NS, Kestenbaum BR, Robinson-Cohen C, Heagerty PJ, Tuttle K, et al. Diabetes and CKD in the United States population, 2009-2014. Clin J Am Soc Nephrol 2017;12:1984-90.

2. Thomas MC, Cooper ME, Zimmet P. Changing epidemiology of type 2 diabetes mellitus and associated chronic kidney disease. Nat Rev Nephrol 2016;12:73-81.

3. Thomas MC, Brownlee M, Susztak K, Sharma K, JandeleitDahm KA, Zoungas S, et al. Diabetic kidney disease. Nat Rev Dis Primers 2015;1:15018.

4. Reidy K, Kang HM, Hostetter T, Susztak K. Molecular mechanisms of diabetic kidney disease. J Clin Invest 2014; 124:2333-40.

5. Stratton IM, Adler AI, Neil HA, Matthews DR, Manley SE, Cull CA, et al. Association of glycaemia with macrovascular and microvascular complications of type 2 diabetes (UKPDS 35): prospective observational study. BMJ 2000;321:40512.

6. UK Prospective Diabetes Study (UKPDS) Group. Intensive blood-glucose control with sulphonylureas or insulin compared with conventional treatment and risk of complications in patients with type 2 diabetes (UKPDS 33). Lancet 1998; 352:837-53.

7. Prischl FC, Wanner C. Renal outcomes of antidiabetic treatment options for type 2 diabetes: a proposed MARE definition. Kidney Int Rep 2018;3:1030-8.

8. Mulvihill EE, Drucker DJ. Pharmacology, physiology, and mechanisms of action of dipeptidyl peptidase-4 inhibitors. Endocr Rev 2014;35:992-1019.

9. Muskiet MH, Smits MM, Morsink LM, Diamant M. The gut-renal axis: do incretin-based agents confer renoprotection in diabetes? Nat Rev Nephrol 2014;10:88-103.

10. Kang YM, Jung CH. Effects of incretin-based therapies on diabetic microvascular complications. Endocrinol Metab (Seoul) 2017;32:316-25.

11. Mosenzon O, Leibowitz G, Bhatt DL, Cahn A, Hirshberg B, Wei $\mathrm{C}$, et al. Effect of saxagliptin on renal outcomes in the SAVOR-TIMI 53 trial. Diabetes Care 2017;40:69-76.
12. Cornel JH, Bakris GL, Stevens SR, Alvarsson M, Bax WA, Chuang LM, et al. Effect of sitagliptin on kidney function and respective cardiovascular outcomes in type 2 diabetes: outcomes from TECOS. Diabetes Care 2016;39:2304-10.

13. Scirica BM, Bhatt DL, Braunwald E, Steg PG, Davidson J, Hirshberg B, et al. Saxagliptin and cardiovascular outcomes in patients with type 2 diabetes mellitus. N Engl J Med 2013;369:1317-26.

14. Green JB, Bethel MA, Armstrong PW, Buse JB, Engel SS, Garg J, et al. Effect of sitagliptin on cardiovascular outcomes in type 2 diabetes. N Engl J Med 2015;373:232-42.

15. Groop PH, Cooper ME, Perkovic V, Hocher B, Kanasaki K, Haneda M, et al. Linagliptin and its effects on hyperglycaemia and albuminuria in patients with type 2 diabetes and renal dysfunction: the randomized MARLINA-T2D trial. Diabetes Obes Metab 2017;19:1610-9.

16. Liberati A, Altman DG, Tetzlaff J, Mulrow C, Gotzsche PC, Ioannidis JP, et al. The PRISMA statement for reporting systematic reviews and meta-analyses of studies that evaluate health care interventions: explanation and elaboration. Ann Intern Med 2009;151:W65-94.

17. Higgins JP, Green S. Cochrane handbook for systematic reviews of interventions. Chichester: John Wiley \& Sons Ltd.; 2008. Chapter 7, Selecting studies and collecting data; $p$. 151-85.

18. Higgins JP, Green S. Cochrane handbook for systematic reviews of interventions. Chichester: John Wiley \& Sons Ltd.; 2008. Chapter 8, Assessing risk of bias in included studies; p. 187-242.

19. Higgins JP, Green S. Cochrane handbook for systematic reviews of interventions. Chichester: John Wiley \& Sons Ltd.; 2008. Chapter 9, Analysing data and undertaking meta-analyses; p. 243-96.

20. Cooper H, Hedges LV, Valentine JC. The handbook of research synthesis and meta-analysis. 2nd ed. New York: Russell Sage Foundation; 2009. Chapter 12, Effect sizes for continuous data; p. 221-35.

21. Egger M, Davey Smith G, Schneider M, Minder C. Bias in meta-analysis detected by a simple, graphical test. BMJ 1997;315:629-34.

22. Sterne JA, Egger M. Funnel plots for detecting bias in metaanalysis: guidelines on choice of axis. J Clin Epidemiol 2001; 54:1046-55.

23. Groop PH, Cooper ME, Perkovic V, Emser A, Woerle HJ, von Eynatten M. Linagliptin lowers albuminuria on top of recommended standard treatment in patients with type 2 dia-

Copyright (C) 2019 Korean Endocrine Society 
betes and renal dysfunction. Diabetes Care 2013;36:3460-8.

24. Taskinen MR, Rosenstock J, Tamminen I, Kubiak R, Patel S, Dugi KA, et al. Safety and efficacy of linagliptin as add-on therapy to metformin in patients with type 2 diabetes: a randomized, double-blind, placebo-controlled study. Diabetes Obes Metab 2011;13:65-74.

25. Del Prato S, Barnett AH, Huisman H, Neubacher D, Woerle HJ, Dugi KA. Effect of linagliptin monotherapy on glycaemic control and markers of $\beta$-cell function in patients with inadequately controlled type 2 diabetes: a randomized controlled trial. Diabetes Obes Metab 2011;13:258-67.

26. Owens DR, Swallow R, Dugi KA, Woerle HJ. Efficacy and safety of linagliptin in persons with type 2 diabetes inadequately controlled by a combination of metformin and sulphonylurea: a 24-week randomized study. Diabet Med 2011; 28:1352-61.

27. Haak T, Meinicke T, Jones R, Weber S, von Eynatten M, Woerle HJ. Initial combination of linagliptin and metformin improves glycaemic control in type 2 diabetes: a randomized, double-blind, placebo-controlled study. Diabetes Obes Metab 2012;14:565-74.

28. Mori H, Okada Y, Arao T, Tanaka Y. Sitagliptin improves albuminuria in patients with type 2 diabetes mellitus. J Diabetes Investig 2014;5:313-9.

29. Nomoto H, Miyoshi H, Sugawara H, Ono K, Yanagiya S, Oita $\mathrm{M}$, et al. A randomized controlled trial comparing the effects of dapagliflozin and DPP-4 inhibitors on glucose variability and metabolic parameters in patients with type 2 diabetes mellitus on insulin. Diabetol Metab Syndr 2017;9:54.

30. DeFronzo RA, Lewin A, Patel S, Liu D, Kaste R, Woerle HJ, et al. Combination of empagliflozin and linagliptin as second-line therapy in subjects with type 2 diabetes inadequately controlled on metformin. Diabetes Care 2015;38:384-93.

31. Lavalle-Gonzalez FJ, Januszewicz A, Davidson J, Tong C, Qiu R, Canovatchel W, et al. Efficacy and safety of canagliflozin compared with placebo and sitagliptin in patients with type 2 diabetes on background metformin monotherapy: a randomised trial. Diabetologia 2013;56:2582-92.

32. Lewin A, DeFronzo RA, Patel S, Liu D, Kaste R, Woerle $\mathrm{HJ}$, et al. Initial combination of empagliflozin and linagliptin in subjects with type 2 diabetes. Diabetes Care 2015;38:394402.

33. Schernthaner G, Gross JL, Rosenstock J, Guarisco M, Fu M, Yee J, et al. Canagliflozin compared with sitagliptin for patients with type 2 diabetes who do not have adequate glycemic control with metformin plus sulfonylurea: a 52-week randomized trial. Diabetes Care 2013;36:2508-15.

34. Tonneijck L, Smits MM, Muskiet MH, Hoekstra T, Kramer MH, Danser AH, et al. Renal effects of DPP-4 inhibitor sitagliptin or GLP-1 receptor agonist liraglutide in overweight patients with type 2 diabetes: a 12-week, randomized, doubleblind, placebo-controlled trial. Diabetes Care 2016;39:204250.

35. Takihata M, Nakamura A, Tajima K, Inazumi T, Komatsu Y, Tamura H, et al. Comparative study of sitagliptin with pioglitazone in Japanese type 2 diabetic patients: the COMPASS randomized controlled trial. Diabetes Obes Metab 2013;15:45562.

36. Nomoto H, Miyoshi H, Furumoto T, Oba K, Tsutsui H, Inoue A, et al. A randomized controlled trial comparing the effects of sitagliptin and glimepiride on endothelial function and metabolic parameters: Sapporo Athero-Incretin Study 1 (SAIS1). PLoS One 2016;11:e0164255.

37. Zografou I, Sampanis C, Gkaliagkousi E, Iliadis F, Papageorgiou A, Doukelis $\mathrm{P}$, et al. Effect of vildagliptin on hsCRP and arterial stiffness in patients with type 2 diabetes mellitus. Hormones (Athens) 2015;14:118-25.

38. Nakamura K, Oe H, Kihara H, Shimada K, Fukuda S, Watanabe K, et al. DPP-4 inhibitor and alpha-glucosidase inhibitor equally improve endothelial function in patients with type 2 diabetes: EDGE study. Cardiovasc Diabetol 2014;13:110.

39. White WB, Cannon CP, Heller SR, Nissen SE, Bergenstal RM, Bakris GL, et al. Alogliptin after acute coronary syndrome in patients with type 2 diabetes. N Engl J Med 2013;369:132735.

40. Yoon SA, Han BG, Kim SG, Han SY, Jo YI, Jeong KH, et al. Efficacy, safety and albuminuria-reducing effect of gemigliptin in Korean type 2 diabetes patients with moderate to severe renal impairment: a 12-week, double-blind randomized study (the GUARD Study). Diabetes Obes Metab 2017;19:590-8.

41. Roden M, Weng J, Eilbracht J, Delafont B, Kim G, Woerle HJ, et al. Empagliflozin monotherapy with sitagliptin as an active comparator in patients with type 2 diabetes: a randomised, double-blind, placebo-controlled, phase 3 trial. Lancet Diabetes Endocrinol 2013;1:208-19.

42. Cooper ME, Perkovic V, McGill JB, Groop PH, Wanner C, Rosenstock J, et al. Kidney disease end points in a pooled analysis of individual patient-level data from a large clinical trials program of the dipeptidyl peptidase 4 inhibitor linagliptin in type 2 diabetes. Am J Kidney Dis 2015;66:441-9.

43. Alter ML, Ott IM, von Websky K, Tsuprykov O, Sharkovska Y, Krause-Relle K, et al. DPP-4 inhibition on top of angio- 
tensin receptor blockade offers a new therapeutic approach for diabetic nephropathy. Kidney Blood Press Res 2012;36: 119-30.

44. Kodera R, Shikata K, Takatsuka T, Oda K, Miyamoto S, Kajitani N, et al. Dipeptidyl peptidase-4 inhibitor ameliorates early renal injury through its anti-inflammatory action in a rat model of type 1 diabetes. Biochem Biophys Res Commun 2014;443:828-33.

45. Sharkovska Y, Reichetzeder C, Alter M, Tsuprykov O, Bachmann S, Secher T, et al. Blood pressure and glucose independent renoprotective effects of dipeptidyl peptidase-4 inhibition in a mouse model of type- 2 diabetic nephropathy. J Hypertens 2014;32:2211-23.

46. Jung GS, Jeon JH, Choe MS, Kim SW, Lee IK, Kim MK, et al. Renoprotective effect of gemigliptin, a dipeptidyl peptidase-4 inhibitor, in streptozotocin-induced type 1 diabetic mice. Diabetes Metab J 2016;40:211-21.

47. Kanasaki K, Shi S, Kanasaki M, He J, Nagai T, Nakamura Y, et al. Linagliptin-mediated DPP-4 inhibition ameliorates kidney fibrosis in streptozotocin-induced diabetic mice by inhibiting endothelial-to-mesenchymal transition in a therapeutic regimen. Diabetes 2014;63:2120-31.

48. Takashima S, Fujita H, Fujishima H, Shimizu T, Sato T, Morii $\mathrm{T}$, et al. Stromal cell-derived factor-1 is upregulated by dipeptidyl peptidase-4 inhibition and has protective roles in progressive diabetic nephropathy. Kidney Int 2016;90:78396.

49. Lovshin JA, Rajasekeran H, Lytvyn Y, Lovblom LE, Khan S, Alemu R, et al. Dipeptidyl peptidase 4 inhibition stimulates distal tubular natriuresis and increases in circulating SDF$1 \alpha(1-67)$ in patients with type 2 diabetes. Diabetes Care 2017; 40:1073-81.

50. Cherney DZ, Perkins BA, Soleymanlou N, Maione M, Lai V, Lee A, et al. Renal hemodynamic effect of sodium-glucose cotransporter 2 inhibition in patients with type 1 diabetes mellitus. Circulation 2014;129:587-97.

51. Rieg T, Gerasimova M, Murray F, Masuda T, Tang T, Rose $\mathrm{M}$, et al. Natriuretic effect by exendin-4, but not the DPP-4 inhibitor alogliptin, is mediated via the GLP-1 receptor and preserved in obese type 2 diabetic mice. Am J Physiol Renal Physiol 2012;303:F963-71.

52. Yang L, Yuan J, Zhou Z. Emerging roles of dipeptidyl peptidase 4 inhibitors: anti-inflammatory and immunomodulatory effect and its application in diabetes mellitus. Can J Diabetes 2014;38:473-9.

53. Shinjo T, Nakatsu Y, Iwashita M, Sano T, Sakoda H, Ishihara $\mathrm{H}$, et al. DPP-IV inhibitor anagliptin exerts anti-inflammatory effects on macrophages, adipocytes, and mouse livers by suppressing NF- $\mathrm{BB}$ activation. Am J Physiol Endocrinol Metab 2015;309:E214-23.

54. Zhuge F, Ni Y, Nagashimada M, Nagata N, Xu L, Mukaida N, et al. DPP-4 inhibition by linagliptin attenuates obesity-related inflammation and insulin resistance by regulating M1/M2 macrophage polarization. Diabetes 2016;65:2966-79.

55. Kubota Y, Miyamoto M, Takagi G, Ikeda T, KirinokiIchikawa S, Tanaka K, et al. The dipeptidyl peptidase-4 inhibitor sitagliptin improves vascular endothelial function in type 2 diabetes. J Korean Med Sci 2012;27:1364-70. 


\section{Appendix 1. Study protocol}

1. Title: Effects of DPP-4 inhibitors on renal outcomes in patients with type 2 diabetes: a systematic review and meta-analysis of randomized controlled trials.

2. Objectives: This study is conducted to investigate the effects of dipeptidyl peptidase-4 (DPP-4) inhibitors on renal outcomes in patients with type 2 diabetes (T2D) compared with placebo or other antidiabetic agents.

3. Protocol and registration: Methods of database search, study selection, data extraction, assessment of study quality and risk of bias, and statistical analysis are prespecified in the protocol at the beginning of the study.

4. Reporting: This systematic review and meta-analysis was reported according to the Preferred Reporting Items for Systematic Review and Meta-Analysis (PRISMA) statement.

\section{Eligible criteria}

1) Study characteristics

(1) Population: patients with T2D

(2) Intervention: DPP-4 inhibitors

(3) Comparison: placebo or other antidiabetic agents

(4) Outcomes of interests

(1) Changes in urine albumin-to-creatinine ratio (UACR) from baseline

(2) Changes in estimated glomerular filtration rate (eGFR) from baseline

(3) Development of microalbuminuria

(4) Development of macroalbuminuria

(5) Regression of albuminuria, defined as the development of normoalbuminuria from microalbuminuria or macroalbuminuria; development of microalbuminuria from macroalbuminuria

(6) Development of end-stage renal disease (ESRD), defined as initiation of renal replacement therapy, renal transplantation, serum creatinine levels $>6.0 \mathrm{mg} / \mathrm{dL}$, or renal failure

(5) Study design: randomized controlled trials (RCTs)

(6) Length of follow-up: at least 12 weeks of study duration

2) Report characteristics

(1) Years considered: published from inception to September 2017

(2) Language: no limitation of language

(3) Publication status: full-text articles with no limitation of publication status

3) Inclusion and exclusion criteria

(1) The study population was men and women with T2D.

(2) We only included RCTs comparing efficacy or safety of DPP-4 inhibitors with those of placebo or other antidiabetic agents in patients with $\mathrm{T} 2 \mathrm{D}$.

(3) Any concurrent use of antidiabetic agents other than glucagon-like peptide-1 receptor agonists was allowed.

(4) Only RCTs with a study duration of 12 weeks or more were included.

(5) We only included RCTs reporting at least one renal outcome including UACR, eGFR, microalbuminuria, macroalbuminuria, doubling of serum creatinine levels, renal failure, ESRD, renal replacement therapy, dialysis, and kidney transplantation.

(6) In case of duplicates or extensions, we only included a study with the longer duration or more information about renal outcomes.

(7) Pooled analysis or secondary analysis was included only when it provided more information about renal outcomes than original publications.

(8) There is no restriction of language.

(9) We included full-text articles with no restriction of publication status.

6. Information sources: We searched electronic databases of MEDLINE, Embase, and the Cochrane Central Register of Controlled Trials. 
7. Search strategy: RCTs of DPP-4 inhibitors in patients T2D were searched using following search terms.

1) MEDLINE: DPP-4 inhibitor OR DPP4 inhibitor OR alogliptin OR anagliptin OR evogliptin OR gemigliptin OR linagliptin OR omarigliptin OR saxagliptin OR sitagliptin OR teneligliptin OR trelagliptin OR vildagliptin

2) Embase: DPP-4 inhibitor OR DPP4 inhibitor OR alogliptin OR anagliptin OR evogliptin OR gemigliptin OR linagliptin OR omarigliptin OR saxagliptin OR sitagliptin OR teneligliptin OR trelagliptin OR vildagliptin

3) The Cochrane Central Register of Controlled Trials: DPP-4 inhibitor OR DPP4 inhibitor OR alogliptin OR anagliptin OR evogliptin OR gemigliptin OR linagliptin OR omarigliptin OR saxagliptin OR sitagliptin OR teneligliptin OR trelagliptin OR vildagliptin

8. Study selection: All identified records were screened and evaluated for eligibility by two reviewers independently. We reviewed titles, abstracts, and full texts of the studies thoroughly. Any disagreements were resolved by consensus among investigators of this study.

9. Data extraction: Standardized data extraction was performed by two reviewers independently as follows. Any discrepancies were resolved by consensus among investigators of this study.
1) First author
2) Publication year
3) Number of randomized participants
4) Mean age of randomized participants, years
5) Study duration, weeks
6) Intervention including names and doses of DPP-4 inhibitors
7) Comparison including placebo or names and doses of other antidiabetic agents
8) Background antidiabetic agents
9) Baseline eGFR, $\mathrm{mL} / \mathrm{min} / 1.73 \mathrm{~m}^{2}$
10) Mean changes in UACR with their standard deviation, $\mathrm{mg} / \mathrm{g}$
11) Mean changes in eGFR with their standard deviation, $\mathrm{mL} / \mathrm{min} / 1.73 \mathrm{~m}^{2}$
12) Number of participants reporting the development of microalbuminuria
13) Number of participants reporting the development of macroalbuminuria
14) Number of participants reporting regression of albuminuria
15) Number of participants reporting the development of ESRD

10. Assessment of study quality and risk bias: We assessed quality and risk of bias of included studies using the Cochrane Risk of Bias Tool. Two reviewers independently evaluated each study based on following aspects of trials:

1) Random sequence generation

2) Allocation concealment

3) Blinding

4) Incomplete outcome data

5) Selective reporting

6) Other sources of bias

\section{Data synthesis}

1) Statistical analysis: We calculated standard mean differences for UACR, weighted mean differences (WMDs) for eGFR with 95\% confidence intervals (CIs), and calculate relative risks (RRs) with $95 \%$ CI for dichotomous variables. In meta-analysis, we used a random-effects model to evaluate WMDs, RRs, and their 95\% CIs.

2) Subgroup analysis: We performed a subgroup analysis for evaluating changes in UACR according to sodium-glucose cotransporter 2 (SGLT2) inhibitors versus non-SGLT2 inhibitors in the control group.

3) Identifying and measuring statistical heterogeneity: We used Cochran's Q test for testing statistical heterogeneity and the $I^{2}$ statistic for measuring the degree of heterogeneity. 